\title{
Magnitude of screening for gestational diabetes mellitus in an urban setting in Tanzania; a cross-sectional analytic study
}

\author{
Akampa Mukuve $^{1^{*}}$ D, Mariam Noorani ${ }^{2}$ Ibrahim Sendagire ${ }^{3}$ and Miriam Mgonja ${ }^{4}$
}

\begin{abstract}
Background: Medical screening detects risk factors for disease or presence of disease in otherwise well persons in order to intervene early and reduce morbidity and mortality. During antenatal care (ANC) it is important to detect conditions that complicate pregnancy, like gestational diabetes mellitus (GDM). Despite international and local guidelines recommending screening for GDM during ANC, there is evidence to suggest that the practice was not being carried out adequately. A major challenge may be lack of consensus on uniform GDM screening and diagnostic guidelines internationally and locally.

The primary objective was to determine the magnitude of screening for GDM among women receiving ANC at the Aga Khan Hospital, Dar es Salaam and Muhimbili National Hospital, Dar es Salaam. Secondary objectives were: to determine the methods used by health practitioners to screen for GDM, to determine the magnitude of undiagnosed gestational diabetes mellitus among women attending ANC and factors associated with screening for GDM among these women.
\end{abstract}

Methods: A cross-sectional analytical study was done. Data collection was done using pre-tested questionnaires and reviewing antenatal care records. The proportion of women attending ANC who were screened for GDM was determined. The $75 \mathrm{~g}$ Oral Glucose Tolerance Test (OGTT) was offered to women who had not been screened after education and consent.

Results: Only 107 out of 358 (29.9\%) had been offered some form of GDM screening. Tests used for GDM screening were random blood sugar (56.8\%), fasting blood sugar (32.8\%), HbA1C (6\%) and $75 \mathrm{~g} O G T$ (3.4\%). The uptake of the OGTT was 27\%. Of these women the prevalence of GDM was 27.9\%. Factors associated with screening for GDM were history of big baby, history of pregnancy induced hypertension and participant awareness of GDM (all p: <0.05).

Conclusions: Screening for GDM among women attending ANC was lower than the World Health Organization target. Efforts should be directed towards promoting GDM screening, increasing awareness about GDM and developing more effective screening methods.

Keywords: Tanzania, Hyperglycaemia in pregnancy, Screening

* Correspondence: akampamukuvem@gmail.com

'Department of Family Medicine, Post Graduate Medical Education, Aga

Khan University, Dar es Salaam, Tanzania

Full list of author information is available at the end of the article

C C The Author(s). 2020 Open Access This article is licensed under a Creative Commons Attribution 4.0 International License, which permits use, sharing, adaptation, distribution and reproduction in any medium or format, as long as you give appropriate credit to the original author(s) and the source, provide a link to the Creative Commons licence, and indicate if changes were made. The images or other third party material in this article are included in the article's Creative Commons licence, unless indicated otherwise in a credit line to the material. If material is not included in the article's Creative Commons licence and your intended use is not permitted by statutory regulation or exceeds the permitted use, you will need to obtain permission directly from the copyright holder. To view a copy of this licence, visit http://creativecommons.org/licenses/by/4.0/ The Creative Commons Public Domain Dedication waiver (http://creativecommons.org/publicdomain/zero/1.0/) applies to the data made available in this article, unless otherwise stated in a credit line to the data. 


\section{Background}

Medical screening identifies apparently healthy people who have a disease or increased risk of disease [1]. The strategy helps clinicians to detect unrecognized symptomatic and pre-symptomatic disease. Screening for conditions therefore enables early diagnosis and intervention which improves clinical outcomes and reduces costs of health care.

Gestational diabetes mellitus (GDM) is diabetes that is first diagnosed in the second or third trimester of pregnancy that is not clearly either preexisting type 1 or type 2 diabetes [2]. GDM is one of the conditions which should be screened for during antenatal care (ANC). This is because GDM increases risk of maternal and fetal complications during pregnancy [3] and adverse health outcomes in the future [4].

The prevalence of GDM varies across populations, ranging from 10.4 to $25 \%$ across the world [5]. Over $90 \%$ of cases occur in low and middle income countries [6]. Although there is a lot of literature on the prevalence, management and complications of GDM, little focus has been put on how much GDM screening i.e. the magnitude of GDM screening is actually done. A study done in Uganda, Tanzania and Burkina Faso showed that health care workers did not provide all required services during ANC despite good attendance [7]. It is worth also noting that guidelines for screening for GDM vary across the globe and within countries [8]. In Tanzania the national guidelines [9] vary from the Tanzania diabetic association [10] guidelines and both vary from the World Health Organization (WHO) guidelines [3]. Tests used to screen for GDM in these national guidelines include the oral glucose tolerance test (OGTT), random blood sugar (RBS), fasting blood sugar (FBS) and glycated haemoglobin (HbA1C).

Lack of vigilance to screen for GDM and varying guidelines may be responsible for poor GDM screening practices in low resource settings as shown in Cameroon [11]. From other studies, some factors associated with screening for GDM include: education status/ literacy, husband support, family support, awareness of GDM or its risks, health care providers informing the women about the test and help with household work [12], marital status, women's autonomy [13], employment status [14], private health insurance status, distance to hospital, journey time to health facility, mode of transport and ability to pay for the service/ financial status [15].

Given the significance of the unfavourable pregnancy outcomes of GDM but paucity of data on how much screening is done for the condition, this study sought to determine the magnitude of screening for GDM among women attending ANC at two tertiary hospitals. The study also determined the tests used for GDM screening during antenatal care, prevalence of undiagnosed GDM and factors associated with screening for GDM.

\section{Methods}

\section{Design and study site}

A cross-sectional analytic study was done at the Aga Khan Hospital Dar es Salaam and Muhimbili National Hospital, Dar es Salaam in Tanzania. The Aga Khan Hospital is a not for profit, tertiary care facility and the biggest private hospital in Tanzania. Its antenatal care clinic attends to averagely 300 women a week. Muhimbili hospital is the national referral hospital providing tertiary care services to referral cases and the population surrounding it. Antenatal care attendance in this hospital averages 1000 women per week.

\section{Participants}

Total sample size was 358. Sample size was determined using a standard formula on the basis of $22 \%$ magnitude of screening for GDM from a study in a similar setting [11], $20 \%$ precision, $80 \%$ power, $5 \%$ non-response rate and type I error of $5 \%$. Each of the 2 study sites provided half of the total sample. Convenient, consecutive sampling was used to achieve the required sample.

The study population comprised of pregnant women above 18 years of age, who had already received some antenatal care and who were above the gestational age at which screening for GDM is routinely performed. Included were pregnant women attending the antenatal care specialist clinics, aged 18 years and above, possession of antenatal card, gestational age of 30 or more weeks calculated using menstrual dates or earliest ultra sound scan, and who attended a 1st or 2nd trimester antenatal care visit at the study site. Women were excluded if they had pre-existing self-reported diagnosis of diabetes mellitus, severe illness, mental illness, participated in another study or were taking corticosteroids, antidepressants, diuretics or beta blockers.

\section{Data collection}

During the routine health talks all women were educated about GDM and given details about the study. Informed, written and signed consent was obtained from willing participants. A mini check list confirmed eligibility of participants.

Data collection tools were an interviewer-administered questionnaire and data extraction sheet. Participants were interviewed using a pre-tested, structured questionnaire in the language of choice between Kiswahili and English. The questionnaire identified patient demographic characteristics, risk factors for GDM, socioeconomic factors associated with screening for GDM, past obstetric and medical history and tests for glycemic status and related information regarding screening for GDM. Additional information including weight, height, body mass index, blood pressure was obtained and verified from the antenatal cards, patient files and laboratory 
reports. This information was recorded on the data collection sheet. Where information from the interview and patient records differed, the latter were used.

Women were considered screened for GDM if WHO 2013 guidelines [3], Tanzania Diabetic association guidelines [10] or Tanzania ministry of health guidelines 2017 [9] had been followed. All participants found not to have been screened for GDM were offered the $75 \mathrm{~g}$ OGTT. The glucometer SD GlucoNavii ${ }^{\circ}$ GDH blood glucose monitoring system and SD BIOSENSOR INC lancets were used. This apparatus had a coefficient of variation $5 \%$ above $5.55 \mathrm{mmol} / \mathrm{l}$ and within standard deviation 4 $\mathrm{mg} / \mathrm{dl}$ for readings below $5.55 \mathrm{mmol} / \mathrm{l}$. For those with medical insurance, the cost of the test was covered by the insurer. Cash paying clients were offered a discounted price at the Aga Khan hospital and it was offered free of charge for those who could not afford. Interpretation of results was according to WHO 2013 guidelines [3]. Women diagnosed with GDM or prediabetic states were informed and referred for appropriate care.

Data were collected on possible determinants of screening for GDM which were either socio-economic factors or risk factors of GDM. The socio-economic factors included: education status/ literacy, marital status, employment status, women's autonomy, private health insurance status, distance to hospital, journey time to health facility, mode of transport, ability to pay for the service/ financial status, husband support, family support, obstetric history, parity, awareness of GDM or its risks, health care providers informing the women about the test and help with household work.

The risk factors for GDM included: glycosuria, Body Mass Index (BMI) $>25$ at time of interview, hypertension in current pregnancy, history of chronic hypertension, history of pregnancy induced hypertension (PIH), history of GDM, history of pre-diabetic states, family history of hypertension, family history of diabetes mellitus, history of multiple pregnancy, history of delivering a macrosomic ( $\geq 4 \mathrm{~kg}$ of weight) baby, clinical or ultrasound diagnosis of large for gestational age in the ongoing pregnancy, history of excessive weight gain $(\geq 5 \mathrm{~kg})$ since 18 years of age, race, history of pregnancy loss and grand-multiparity ( $\geq 5$ pregnancies).

\section{Data analysis}

Data was entered into Microsoft excel 2007 and cleaned. The data was then transferred to SPSS 20 for analysis. We defined "magnitude" of screening for GDM as the proportion of participants attending ANC who had received any test aimed at screening for GDM. Various tests done during screening were categorized and frequencies and percentages for each calculated and recorded. The percentage of participants who had not initially screened but were diagnosed with GDM by OGTT in the study was the magnitude of undiagnosed GDM. Socio-economic determinants of screening for GDM and risk factors for GDM were evaluated for association with screening for GDM using the chi-square test and associated $p$-values. Factors found to be statistically significant by chi square test were further analyzed using logistic regression for possible association with screening for GDM.

\section{Results}

The study period was July to September 2018. During the study period July to September 2018, there were 2250 women who attended specialist ANC at the study sites. As shown in Fig. 1, approximately 1500 were eligible to participate. Women who consented were interviewed. Six women were excluded. Two had overt diabetes while four had participated in other studies.

Demographic and obstetric characteristics are summarized in Table 1. The mean age of the participants was 30.2 years (SD: 4.8; range 20-44). Most of the participants were between 20 and 30 years, accounting for $57.6 \%$ of the study population. The mean gestational age was 34.9 weeks of gestation ( $\mathrm{SD}=3.1$; range $30-42)$. Of all the participants, $97.2 \%$ were black African, while $2.8 \%$ were of Asian or of other origins.

\section{Magnitude of screening for GDM}

The overall magnitude of screening for GDM at both hospitals was $29.9 \%$ (107 of 358). The magnitude was 21.5\% (38 of 177) at Aga Khan Hospital and $38.1 \%$ at Muhimbili National Hospital (69 of 181). Of the 251 of women found not to have been screened for GDM only $27 \%$ (68) accepted the offered OGTT.

\section{Tests used to screen for GDM}

Of the 107 participants screened for GDM, 56.8\% (66) had done random blood sugar (RBS), 32.8\% (38) fasting blood sugar (FBS), 6\% [7] HbA1C and 3.4\% [3] the $75 \mathrm{~g}$ Oral glucose tolerance test (OGTT).

\section{Prevalence of undiagnosed GDM}

The prevalence of undiagnosed GDM was 27.9\% (19 of 68).

\section{Factors associated with screening for GDM}

As shown in Tables 2 and 3, chi square analysis revealed factors with statistically significant association with screening for GDM to be: awareness about GDM $(p<0.001)$, health worker communication about GDM screening $(p<0.001)$, partner support during ANC ( $p=$ $0.045)$, family support during ANC (p 0.049), history of pregnancy induced hypertension (PIH) $(p=0.022)$, history of delivering macrosomic baby $(p=0.023)$ and history of glycosuria $(p=0.002)$. Logistic regression analysis 


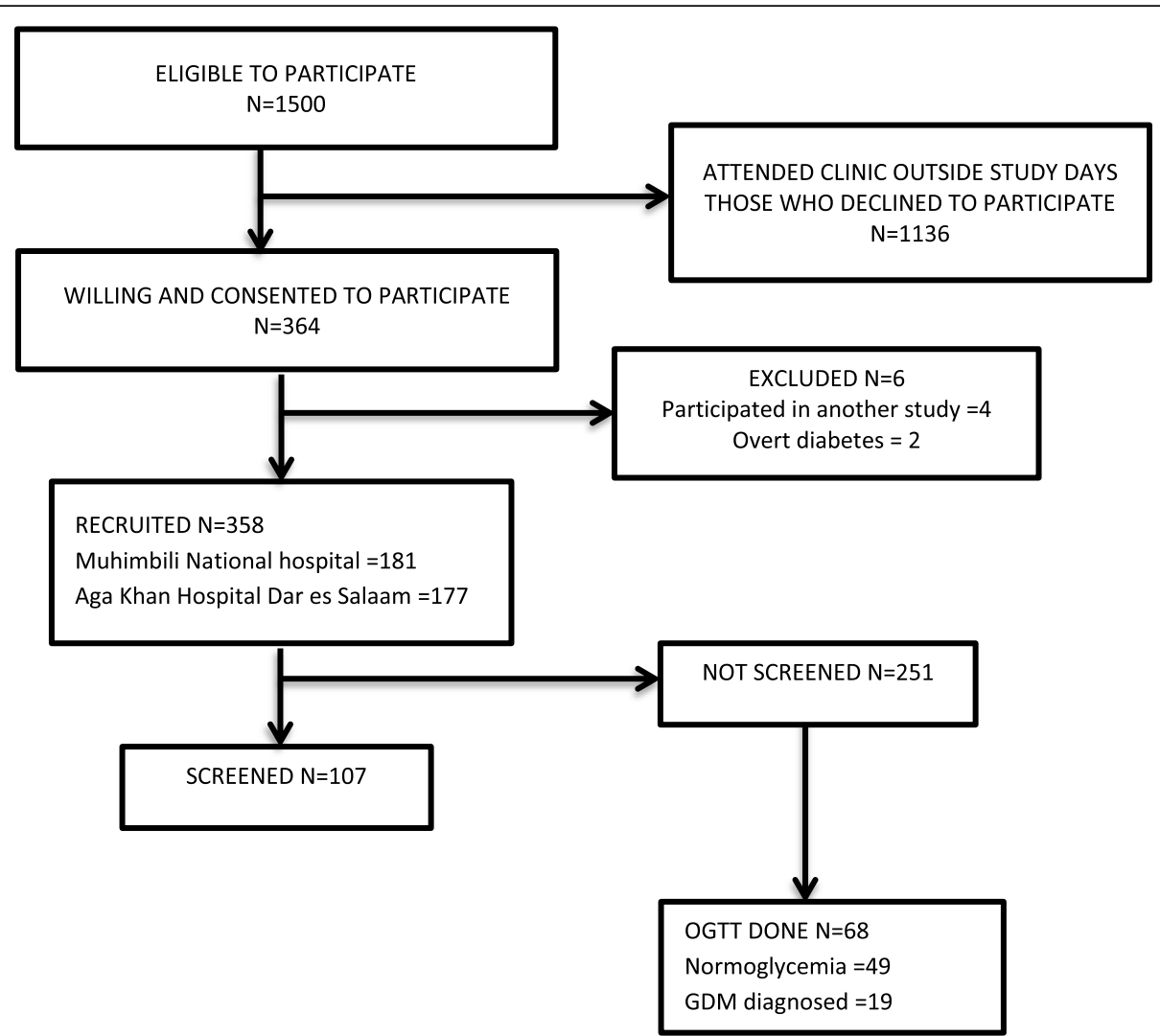

Fig. 1 Study profile of participants attending Antenatal Care at Muhimbili National Hospital and Aga Khan Hospital Dar es Salaam in 2018

(Table 4) revealed that history of delivering a macrosomic baby $(p=<0.001$ OR $2.1595 \%$ CI: $1.12-4.10)$, history of PIH $(p=<0.021$ OR $2.3495 \%$ CI: $1.13-4.82)$ and awareness of GDM $(p=<0.001$ OR 2.79 95\% CI: 1.744.45) were the only 3 factors associated with screening for GDM.

\section{Discussion}

In this study the magnitude of screening for GDM was only $30 \%$. This value is somewhat similar to a Cameroon study which found the screening level at 22\% [11]. Both of these values are lower than the WHO target for a screening strategy which is $70 \%$ of the target population [16]. The study was done in specialist clinics in tertiary facilities, with adequate facilities for screening. In this study specialist clinics were considered because obstetricians were expected to be able to address the complexity and interpretation of different guidelines and tests for GDM better than other cadres. The low screening rate could not be attributed to poor resources or expertise. Another study in the region showed that health workers omit certain practices stipulated in ANC guidelines. Inadequate screening was attributed to limited resources and expertise in the rural settings in that study [7].
The tests used for screening for GDM in order of frequency were RBS, FBS, HbA1C and OGTT. The trend reflects the ease of performing a particular test. Compared to the OGTT the other tests are easy to use, less labour intensive and more accessible [17]. Random blood sugar and HbA1C also do not require fasting [18]. Performing these tests is therefore convenient to both the health worker and the pregnant woman [18]. Whereas the OGTT has better diagnostic accuracy than the other tests, it is more expensive and laborious to perform [18]. This possibly is the reason why it is performed less frequently (3.4\%) than other tests. If we were to use the generally accepted OGTT as the screening test for GDM, the magnitude of screening of $3.4 \%$ is far below the expected standard of care. In Uganda the uptake of the OGTT was shown to be $75.4 \%$ [5]. It would therefore plausible for health workers to offer the OGTT more in this setting.

The prevalence of undiagnosed GDM was 28\%. Prevalence of GDM in a previous Tanzanian study also done in an urban setting was $8.4 \%$ [19]. Just like in our study the $75 \mathrm{~g}$ OGTT was used for diagnosis in that study. Our findings may be suggestive of an increasing prevalence of GDM in Tanzanian urban areas. The prevalence in our study is somewhat similar to $31.9 \%$ in Uganda [5], 
Table 1 Baseline demographic and clinical characteristics of participants attending Antenatal Care at Muhimbili National Hospital and Aga Khan Hospital Dar es Salaam in 2018

\begin{tabular}{cll}
\hline & $\begin{array}{l}\text { Frequency } \\
\text { (Total 358) }\end{array}$ & $\begin{array}{l}\text { Percentage } \\
\text { (Total 100\%) }\end{array}$ \\
\hline Age $^{\text {a }}$ & & \\
$18-25$ & 61 & 17.1 \\
$26-30$ & 135 & 37.9 \\
$31-35$ & 113 & 31.7 \\
$36-40$ & 37 & 10.4 \\
$>40$ & 10 & 2.8
\end{tabular}

\section{Residence}

Ilala

Kinondoni

Ubungo

Temeke

Kigamboni

Outside Dar es salaam

\section{Gravidity}

Prime Gravida

G2

G3

G4

$\geq \mathrm{G} 5$

Number of GDM risk factors

0

1

2

3

4

$\geq 5$

Number of previous ANC visits

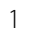

\section{2}

$$
2
$$$$
3
$$$$
4
$$$$
5
$$$$
\geq 6
$$

Gestational age

\begin{tabular}{lll}
$30-31.9$ & 66 & 18.4 \\
$32-34.9$ & 103 & 28.8 \\
$35-37.9$ & 114 & 31.9 \\
$38-40.9$ & 70 & 19.5 \\
$\geq 41$ & 5 & 1.39 \\
\hline
\end{tabular}

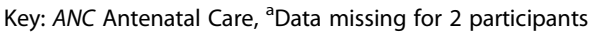

Table 2 Univariable analysis of socio-economic determinants of screening for Gestational Diabetes Mellitus among women attending antenatal clinic at Muhimbili National Hospital and Aga Khan Hospital Dar es Salaam in 2018

\begin{tabular}{lll}
\hline Factor & Prevalence (\%) & $p$-value \\
\hline Educated > tertiary level & 71.8 & 0.926 \\
Married & 82.4 & 0.880 \\
Independent source of income & 79.6 & 0.270 \\
Autonomy & 76.0 & 0.331 \\
Medical insurance & 71.2 & 0.412 \\
Personal vehicle & 27.9 & 0.179 \\
Live in llala & 26.5 & 0.288 \\
Having help with housework & 82.7 & 0.886 \\
Partner ANC attendance & 64.8 & 0.045 \\
Escorted by family member to ANC & 52.8 & 0.049 \\
Awareness of GDM & 45.0 & $<0.001$ \\
Health worker communication & 27.7 & $<0.001$ \\
\hline
\end{tabular}

Key: ANC Antenatal care, GDM Gestational Diabetes Mellitus

$31 \%$ in Cameroon [11] and 25.8\% in south Africa [20]. These findings show a higher burden than the global prevalence of $16 \%$ [21]. Uptake of the OGTT was $27 \%$ which was similar to the $22 \%$ of the Cameroon study. However the uptake varies from $75.4 \%$ found in a Ugandan study [5] and $55.4 \%$ in a South African study [20]. These higher uptake rates could be because in

Table 3 Univariable analysis of risk factors for Gestational Diabetes Mellitus among women attending Antenatal care at Muhimbili National Hospital and Aga Khan Hospital Dar es Salaam in 2018

\begin{tabular}{lll}
\hline Risk factor & Prevalence (\%) & $p$-value \\
\hline Grand multiparity & 6.9 & 0.709 \\
History of GDM & 1.1 & 0.094 \\
History of PIH & 9.5 & 0.022 \\
Hypertension detected in current pregnancy & 2.5 & 0.707 \\
Pre-diabetic state & 2.0 & 0.325 \\
Glycosuria & 3.6 & 0.002 \\
BMI > 25 & 88.5 & 0.619 \\
History of big baby & 12.6 & 0.023 \\
LGA in current pregnancy & 4.5 & 0.903 \\
Excessive weight gain & 65.9 & 0.281 \\
Family history of DM & 29.6 & 0.671 \\
Family history of HT & 43.6 & 0.123 \\
History of multiple pregnancy & 3.7 & 0.479 \\
History of hypertension & 4.5 & 0.662 \\
History of pregnancy loss & 27.9 & 0.587 \\
\hline
\end{tabular}

Key: PIH Pregnancy Induced Hypertension, GDM Gestational Diabetes Mellitus, ANC Antenatal care, HT Hypertension, LGA Large for Gestational Age, BMI Body Mass Index 
Table 4 Multivariate analysis of factors associated with screening for Gestational Diabetes Mellitus among ANC attendees at Muhimbili National Hospital and Aga Khan Hospital Dar es Salaam in 2018

\begin{tabular}{|c|c|c|c|c|}
\hline \multirow[t]{2}{*}{ Characteristic } & \multicolumn{2}{|c|}{ Screened for GDM } & \multirow[t]{2}{*}{ Odds ratio $(95 \% \mathrm{Cl})$} & \multirow[t]{2}{*}{$p$-value } \\
\hline & Yes $=107(\mathrm{~N} / \%)$ & $\mathrm{No}=251(\mathrm{~N} / \%)$ & & \\
\hline \multicolumn{5}{|l|}{ Glycosuria } \\
\hline Yes & $9(8.4 \%)$ & $4(1.6 \%)$ & $0.96(0.880-1.053)$ & 0.401 \\
\hline No & 97 (90.7\%) & $247(98.4 \%)$ & & \\
\hline \multicolumn{5}{|c|}{ History of delivering big baby } \\
\hline Yes & $20(18.7 \%)$ & $25(10 \%)$ & $2.15(1.127-4.101)$ & 0.020 \\
\hline No & 87 (81.3\%) & $226(90 \%)$ & & \\
\hline \multicolumn{5}{|l|}{ History of PIH } \\
\hline Yes & $16(15 \%)$ & $18(7.2 \%)$ & $2.34(1.131-4.827)$ & 0.021 \\
\hline No & $91(85 \%)$ & $233(92.8 \%)$ & & \\
\hline \multicolumn{5}{|c|}{ Awareness of GDM } \\
\hline Yes & $67(62.6 \%)$ & $94(37.5 \%)$ & $2.79(1.749-4.459)$ & $<0.001$ \\
\hline No & $40(37.4 \%)$ & $157(62.5 \%)$ & & \\
\hline \multicolumn{5}{|c|}{ Partner attendance of ANC } \\
\hline Yes & $77(72 \%)$ & $155(61.8 \%)$ & $0.96(0.902-1.038)$ & 0.360 \\
\hline No & $29(27.1 \%)$ & $96(38.2 \%)$ & & \\
\hline \multicolumn{5}{|c|}{ Family member attendance of ANC } \\
\hline Yes & $65(60.7 \%)$ & $124(49.4 \%)$ & $1.57(0.993-2.504)$ & 0.053 \\
\hline No & $42(39.3 \%)$ & $127(50.6 \%)$ & & \\
\hline \multicolumn{5}{|c|}{ Health worker communication about GDM tests } \\
\hline Yes & $91(85 \%)$ & $8(3.2 \%)$ & $1.03(0.996-1.068)$ & 0.087 \\
\hline No & $15(14 \%)$ & $219(87.3 \%)$ & & \\
\hline
\end{tabular}

Key: PIH Pregnancy Induced Hypertension, GDM Gestational Diabetes Mellitus, ANC Antenatal care

these studies, the primary objective was to determine the prevalence of GDM using the OGTT. In our study we offered the OGTT as part of a secondary objective.

Factors found to be statistically significantly associated with screening were awareness about GDM, history of pregnancy induced hypertension (PIH) and history of delivering a macrosomic baby. Awareness of GDM in our study was $45 \%$, which is similar to $40.3 \%$ in a multiethnic Australian study [22] but varies from $17.5 \%$ in an Indian study [23]. Similar to the findings in our study, another study in Tamil, India showed that knowledge of GDM promotes GDM screening [12]. People are more inclined to accept or do tests if they are aware about the condition and its complications. Health literacy enables patients to access and effectively use health information [24], while low health literacy is associated with poor health outcomes [25]. PIH is a mild form of pre-eclampsia and eclampsia which is the leading direct cause of maternal death in Tanzania [26]. This could explain why clinicians are more vigilant with these women. Delivering a macrosomic baby is the commonest complication of GDM in some settings. For example in a
Ugandan study, macrosomia was the only obstetric complication associated with hyperglycemia in pregnancy [5]. This also explains the possible increased vigilance with these women.

\section{Conclusions}

In conclusion, in this study we found a low magnitude of screening for GDM (30\%). This is inadequate compared to the recommended WHO target of $70 \%$. A significant proportion (28\%) of women with GDM had not been diagnosed with the condition; which could account for significant adverse pregnancy outcomes.

A majority of participants (over $75 \%$ ) had attended ANC 4 or more times by the time we evaluated them for screening. Those many contacts with a health worker are a missed opportunity to screen for GDM and to provide quality, comprehensive ANC services. A sizeable proportion of women were not aware of GDM. This calls for wider sensitization on GDM. Universal rather than selective screening for GDM should be adopted in this setting given the high prevalence of risk factors for GDM. A less cumbersome gold standard test for GDM diagnosis and 
screening needs to be developed to facilitate ease of screening. A study of attitudes and practices of ANC providers would dissect further why adequate screening for GDM is not conducted. Studies should be done to determine factors associated with poor uptake of the OGTT in this setting. Follow up studies should be done on interventions to increase screening for GDM.

\section{Generalizability}

This study was hospital based and urban based. Therefore the study findings may not be generalised to all settings.

\section{Limitations}

In our study, patients were consecutively sampled. This method of sampling could have introduced some level of bias in our findings. However, we have no reasons to believe that manner of selecting our sample would be associated with the dependent variable of being screened or not. The principal investigator and the research assistants were not part of the clinical teams offering ANC services.

Observational studies, like ours are prone to social desirability phenomenon while getting responses from study participants. However, the impact of this phenomenon on our study was reduced for most of the time by corroborating the participants' responses with data that was written on the ANC. Our study did not explore the specific laboratory methods of performing the tests used in GDM screening. However, this could not have affected the study results.

Our study did not explore effects of health worker workload, knowledge and cost of test on the magnitude of screening for GDM. However, cost for the tests was either covered by insurance, heavily discounted or offered free of charge by the health services. All ANC was offered by obstetricians who are assumed to have adequate knowledge and similar level of training. We have no reason to believe that the study results could be different if these factors were explored.

\section{Supplementary information}

Supplementary information accompanies this paper at https://doi.org/10. 1186/s12884-020-03115-3.

Additional file 1. Questionnaire.

Additional file 2. Data extraction sheet.

\section{Abbreviations}

ANC: Antenatal care; AKU: Aga Khan University; BMI: Body Mass Index; Cl: Confidence Interval; FBS: Fasting Blood Sugar; GDM: Gestational Diabetes Mellitus; HbA1C: Glycated Haemoglobin; OGTT: Oral Glucose Tolerance Test; OR: Odds Ratio; PIH: Pregnancy Induced Hypertension; RBS: Random Blood Sugar; WHO: World Health Organization

\section{Acknowledgements}

We acknowledge the support of the research participants and the staff of the ANC clinics of Aga Khan Hospital Dar es salaam and Muhimbili National hospital. We thank Mr. James Orwa, Mr. Kabwama Steven and Dr. Shamira

Pira for their guidance in data analysis.

\section{Authors' contributions}

AM conceptualized and designed the study and research questions, carried out data collection, data analysis and initial interpretation, and drafted the manuscript. MM was the content supervisor of the project. IS was the methodology supervisor of the project. MN was a supervisor of the project. MM, IS and MN reviewed and revised the analysed data and manuscript. All authors read and approved the final manuscript.

\section{Funding}

The Aga Khan University (AKU) provided funding towards data collection and a conducive work station for the authors. AKU had no role in study design, active data collection, analysis, interpretation of data and writing the manuscript.

\section{Availability of data and materials}

The datasets analysed during the study are available from the corresponding author on reasonable request.

\section{Ethics approval and consent to participate}

This study was conducted in accordance with the ethical standards of the Helsinki Declaration of the World Medical Association 1964. The research proposal was submitted to the Aga Khan University Research sub-committee for review. Permission to conduct the study was obtained from the Aga Khan Hospital Dar es Salaam and Muhimbili National Referral Hospital research ethics review committees. The study was finally approved by the Tanzanian National Medical Research Institute. Participants gave informed, written and signed consent.

\section{Consent for publication}

Not applicable.

\section{Competing interests}

The authors declare that they have no competing interests.

\section{Author details}

${ }^{1}$ Department of Family Medicine, Post Graduate Medical Education, Aga Khan University, Dar es Salaam, Tanzania. ${ }^{2}$ Department of Obstetrics and Gynecology, Aga Khan University, P.O BOX 38129, Plot 42, Ufukoni Road, Dar es Salaam, Tanzania. ${ }^{3}$ MSF/Epicentre Mbarara Research Centre, Mbarara, Uganda. ${ }^{4}$ Department of Pediatrics and Child Health, Aga Khan University, Dar es Salaam, Tanzania.

Received: 7 October 2019 Accepted: 16 July 2020

Published online: 23 July 2020

\section{References}

1. Armstrong $\mathrm{N}$, Eborall $\mathrm{H}$. The sociology of medical screening: past, present and future. Sociol Health IIIn. 2012:34(2):161-76.

2. Cefalu WT, Berg EG, Saraco M, Petersen MP, Uelmen S, Robinson S. Classification and diagnosis of diabetes: standards of medical Care in Diabetes-2019. Diabetes Care. 2019;42:S13-28.

3. WHO. Diagnostic Criteria and Classification of Hyperglycemia First Detected in Pregnancy. WHO/NMH/MND/132. Geneva: WHO Press; 2013.

4. Shou C, Wei Y-M, Wang C, Yang H-X. Updates in long-term maternal and Fetal adverse effects of gestational diabetes mellitus. Maternal-Fetal Medicine. 2019;1(2):91-4.

5. Nakabuye B, Bahendeka S, Byaruhanga R. Prevalence of hyperglycaemia first detected during pregnancy and subsequent obstetric outcomes at St. Francis Hospital Nsambya. BMC Res Notes. 2017;10(1):174.

6. Mwanri AW, Kinabo J, Ramaiya K, Feskens EJ. Gestational diabetes mellitus in sub-Saharan Africa: systematic review and metaregression on prevalence and risk factors. Tropical Med Int Health. 2015;20(8):983-1002.

7. Conrad P, Schmid G, Tientrebeogo J, Moses A, Kirenga S, Neuhann F, et al. Compliance with focused antenatal care services: do health workers in rural 
Burkina Faso, Uganda and Tanzania perform all ANC procedures? Tropical Med Int Health. 2012;17(3):300-7.

8. Agarwal MM. Gestational diabetes mellitus: an update on the current international diagnostic criteria. World J Diabetes. 2015;6(6):782-91.

9. Ministry of health cd, gender, elderly, and children. Standard treatment guidelines and essential medicines list, Tanzania mainland. Dar es Salaam2017.

10. Tanzania Diabetic Association MoHSWaTDA. Cardiovascular Disease, Type 2 Diabetes, Obesity,Cancer, Chronic Obstructive Pulmonary Disease, and Hyperlipidaemia Care; Case Management Training Modules; NCD Training Manuals 2014. 2014. p. 52-56.

11. Djomhou M. Maternal hyperglycemia during labor and related immediate post-partum maternal and perinatal outcomes at the Yaoundé Central Hospital, Cameroon. J Health Population Nutrit. 2016;35:28.

12. Karoline Kragelund Nielsen TR, Anil Kapur, Peter Damm. Factors influencing timely initiation and completion of gestational diabetes mellitus screening and diagnosis - a qualitative study from Tamil Nadu, India. 2017;17:255.

13. Nisar N, White F. Factors affecting utilization of antenatal care among reproductive age group women (15-49 years) in an urban squatter settlement of Karachi. J Pakistan Med Assoc. 2003;53(2):47-53.

14. Akowuah JA, Agyei-Baffour $P$, Awunyo-Vitor D. Determinants of antenatal healthcare utilisation by pregnant women in third trimester in peri-urban Ghana. J Trop Med. 2018;2018.

15. Dea AO. Screening uptake rates and the clinical and cost effectiveness of screening for gestational diabetes mellitus in primary versus secondary care: study. 2014;15:27.

16. Organization WH. Cancer control: knowledge into action. WHO guide for effective programmes: prevention. Manila: World Health Organization; 2007.

17. Bhavadharini B, Mahalakshmi MM, Maheswari K, Kalaiyarasi G, Anjana RM, Deepa $\mathrm{M}$, et al. Use of capillary blood glucose for screening for gestational diabetes mellitus in resource-constrained settings. Acta Diabetol. 2016;53(1): 91-7.

18. Trujillo J, Vigo A, Reichelt A, Duncan B, Schmidt M. Fasting plasma glucose to avoid a full OGTT in the diagnosis of gestational diabetes. Diabetes Res Clin Pract. 2014;105(3):322-6.

19. Mwanri AW, Kinabo J, Ramaiya K, Feskens EJ. Prevalence of gestational diabetes mellitus in urban and rural Tanzania. Diabetes Res Clin Pract. 2014; 103(1):71-8.

20. Adams $S$, Rheeder P. Screening for gestational diabetes mellitus in a south African population: prevalence, comparison of diagnostic criteria and the role of risk factors. S Afr Med J. 2017;107(6):523-7.

21. Hod M, Kapur A, Sacks DA, Hadar E, Agarwal M, Di Renzo GC, et al. The International Federation of Gynecology and Obstetrics (FIGO) initiative on gestational diabetes mellitus: a pragmatic guide for diagnosis, management, and care. Int J Gynecol Obstet. 2015;131:S173-211.

22. Carolan M, Steele C, Margetts H. Knowledge of gestational diabetes among a multi-ethnic cohort in Australia. Midwifery. 2010;26(6):579-88.

23. Shriraam V, Rani MA, Sathiyasekaran B, Mahadevan S. Awareness of gestational diabetes mellitus among antenatal women in a primary health center in South India. Indian J Endocrinol Metabolism. 2013:17(1):146.

24. Singh SG, Aiken J. The effect of health literacy level on health outcomes in patients with diabetes at a type $v$ health Centre in Western Jamaica. Int J Nurs Sci. 2017:4(3):266-70.

25. Berkman ND, Sheridan SL, Donahue KE, Halpern DJ, Crotty K. Low health literacy and health outcomes: an updated systematic review. Ann Intern Med. 2011;155(2):97-107.

26. Pembe AB, Paulo C, D'Mello BS, van Roosmalen J. Maternal mortality at Muhimbili National Hospital in Dar-es-salaam, Tanzania in the year 2011. BMC Pregnancy Childbirth. 2014;14:320.

\section{Publisher's Note}

Springer Nature remains neutral with regard to jurisdictional claims in published maps and institutional affiliations.

Ready to submit your research? Choose BMC and benefit from:

- fast, convenient online submission

- thorough peer review by experienced researchers in your field

- rapid publication on acceptance

- support for research data, including large and complex data types

- gold Open Access which fosters wider collaboration and increased citations

- maximum visibility for your research: over $100 \mathrm{M}$ website views per year

At $\mathrm{BMC}$, research is always in progress.

Learn more biomedcentral.com/submissions 